\title{
La estructura sistemática del tesauro: indicadores para evaluar su calidad
}

\author{
Ana M. Martínez*, Cristina A. Ristuccia**, Edgardo A. Stubbs ${ }^{\star *}$, \\ Julia C. Valdez ${ }^{\star *}$, Viviana L. Gamba**, Paola V. Mendes**, \\ Carolina Unzurrunzaga**, María L. Caminotti**
}

Resumen: Se proponen cuatro indicadores para evaluar la calidad de la estructura sistemática del tesauro, considerando que las especificaciones incluidas en las normas para su diseño y desarrollo, así como los principios de la teoría de la clasificación son los requisitos de calidad que deben alcanzar las características inherentes de los tesauros. Las características que no cumplen con dichos requisitos se consideran no conformidades. Se proponen cuatro indicadores para medir las no conformidades, que fueron probados en tres tesauros en español (DeCS, Spines y Tesauro de la Unesco). Los resultados, que constituyen no conformidades, fueron: 1) Porcentaje de términos preferentes que representan más de un concepto: 10,8\% para DeCS, 7,3\% Spines y 10,9\% Unesco. 2) Porcentaje de términos preferentes que carecen de relación jerárquica: 0 DeCS, 1,4\% Spines y 1,1\% Unesco. 3) Porcentaje de términos preferentes con dos o más términos genéricos: 53,8\% DeCS, 14,9\% Spines, 0 Unesco. 4) Porcentaje de términos preferentes con un solo término específico: 13,5\% DeCS, 3,5\% Spines, 10,3\% Unesco. Se concluye que estos indicadores cumplen con los criterios de un buen indicador y pueden ser útiles para la evaluación de la calidad de la estructura sistemática del tesauro durante el proceso de diseño y desarrollo.

Palabras clave: calidad, estructura sistemática, indicadores, tesauros.

\section{The systematic structure of a thesaurus: indicators for quality assessment}

Abstract: Four indicators are proposed to assess the quality of a thesaurus' systematic structure. This proposal considers that the specifications included in thesauri design and development standards, together with the principles of classification theory, represent the quality requirements to be met by the inherent characteristics of a thesaurus. The characteristics that do not meet the requirements are considered nonconformities. Four indicators are proposed to measure nonconformities, and were tested in three thesauri in Spanish. The results (i.e., nonconformities) were: 1) Percentage of preferred terms designating more than one concept: 10,8\% for DeCS, 7,3\% Spines and 10,9\% Unesco.

\footnotetext{
* Instituto de Investigaciones en Humanidades y Ciencias Sociales (UNLP-CONICET). Correo-e: ammarti@speedy.com.ar.

** Departamento de Bibliotecología, Facultad de Humanidades y Ciencias de la Educación, Universidad Nacional de La Plata. Argentina.

Recibido: 22-12-2009; 2. ${ }^{a}$ versión: 19-01-2010; aceptado: 21-01-2010.
} 
2) Percentage of preferred terms without a hierarchical relation: O DeCS, 1,4\% Spines and 1,1\% Unesco. 3) Percentage of preferred terms with more than one broader term: 53,8\% DeCS, 14,9\% Spines, O Unesco. 4) Percentage of preferred terms with only one narrower term: 13,5\% DeCS, 3,5\% Spines, 10,3\% Unesco. We conclude that the proposed indicators can be useful for assessing the quality of a thesaurus' systematic structure during the design and development processes.

Keywords: Indicators, quality, systematic structure, thesauri.

\section{Introducción}

La evaluación de los tesauros ha sido tratada por diversos autores, durante largo tiempo, con distintas técnicas. Gil Leiva (2008) sintetiza estos aportes en dos tipos de evaluación:

- Evaluación intrínseca: su objeto de análisis es el tesauro propiamente dicho. Esta evaluación puede ser, a su vez, de dos tipos: a) cualitativa (Lancaster (2002) la denomina superficial), que evalúa si son adecuados aspectos tales como la introducción, el uso de calificadores y notas de alcance, la reciprocidad de las relaciones, las formas gramaticales de los términos preferentes, etc., y b) cuantitativa (Lancaster (2002) la denomina estadística), en la que se pueden aplicar indicadores.

- Evaluación extrínseca: su objeto de análisis es el comportamiento del tesauro en la indización y la recuperación de información.

Desde otro punto de vista, se puede afirmar que hay tres etapas en las que puede ser evaluado un tesauro: la etapa de diseño y desarrollo, la etapa de indización y la etapa de recuperación.

Para la etapa de indización, Aitchison y otros (2000) y Lancaster (2002) coinciden en recomendar un período de prueba durante el cual el tesauro es utilizado por distintos indizadores, en distintas unidades de información, con el fin de obtener la retroalimentación necesaria para realizar ajustes y correcciones en el tesauro. Por su parte, Owens y Cochrane (2004) han propuesto un algoritmo para evaluar el tesauro tanto en la indización como en la recuperación.

Para la recuperación, las mediciones más conocidas son las tasas de precisión y de acierto (Claverdon y Mills, 1985; Foskett, 1996). En el resultado de estas mediciones intervienen distintas variables como la exhaustividad y la especificidad de la indización, la interfaz usuario-sistema, la experiencia de búsqueda de los usuarios finales, etc., pero también se han utilizado para evaluar específicamente la recuperación por términos preferentes, ya sea de un tesauro o de otros vocabularios controlados. La tasa de precisión ha sido incorporada como indicador en la norma ISO 11620:2008 (International Organization for Standardization, 2008), mientras que la tasa de acierto, hoy en día, puede tener una importancia especial para evaluar la búsqueda expandida (entendida como la búsqueda si- 
multánea en varias bases de datos y la Web con los mismos términos), aunque otros autores han propuesto otras formas de evaluar este tipo de búsqueda (Chung y Lee, 2004).

La evaluación del tesauro en las etapas de indización y recuperación coincide con la evaluación extrínseca de Gil Leiva (2008) y escapan al propósito del presente trabajo, que se centra en la evaluación de un aspecto particular del tesauro (su estructura sistemática), mediante el uso de indicadores (evaluación intrínseca estadística), durante la etapa de diseño y desarrollo, es decir antes de que el tesauro sea utilizado por indizadores y usuarios finales.

La estructura sistemática es una de las características propias del tesauro, que lo distingue de otros vocabularios controlados como las listas de términos o las listas de encabezamientos de materia (Aitchison y otros, 2000; Lancaster, 2002; International Organization for Standardization, 1986; BSI Group, 2005a; National Information Standards Organization, 2005). En esa estructura sistemática, todos los términos preferentes del tesauro se encuentran organizados en una forma lógica por principios derivados principalmente de la teoría de la clasificación.

Respecto a los niveles de organización, Aitchison y otros (2000) reconocen: a) un micronivel que se refiere a la relación jerárquica entre dos términos preferentes, y b) un macronivel que se refiere a la relación de cada término preferente con la estructura sistemática general. Las normas para el diseño y desarrollo de tesauros especifican detalladamente cómo establecer las relaciones jerárquicas entre dos términos preferentes o micronivel (International Organization for Standardization, 1986; BSI Group, 2005a; National Information Standards Organization, 2005). En cambio, son muy escuetas al especificar cómo se organizan dichos términos preferentes en facetas o categorías (macronivel), ya que no describen las etapas del proceso de análisis como lo hacen por ejemplo Mills (2004) o Vickery (1960), ni se menciona un solo principio del análisis de facetas (Ranganathan, 1967; Spiteri, 1998).

Tanto las normas como distintos autores sugieren el análisis de facetas como un método para generar la estructura sistemática completa (Aitchison y otros, 2000; Lancaster, 2002). Otros autores consideran que el análisis de facetas no solo es un método para hacerlo, sino el único método (Mills, 2004). Como quiera que sea, no es adecuado desarrollar la estructura sistemática de un tesauro sin recurrir a los principios de la teoría de la clasificación, definidos por figuras tales como Ranganathan y los miembros del Classification Research Group (CRG) de Gran Bretaña (Mills, 2004; Vickery, 1960; Ranganathan, 1967).

A pesar de lo antes expuesto, la experiencia indica que muchos tesauros no cumplen estrictamente con las especificaciones de las normas y los principios de la teoría de la clasificación, motivo por el cual es necesario evaluar la calidad de su estructura sistemática.

La norma ISO 9000:2005 (International Organization for Standardization, 2005) define calidad como "el grado en el que un conjunto de características inherentes cumple con los requisitos". A su vez, una característica inherente es un "ras- 
go diferenciador" que "existe en algo [producto, proceso, sistema], especialmente como una característica permanente". Por último, requisito se define como "la necesidad o expectativa establecida, generalmente implícita u obligatorian. De modo que si las características inherentes cumplen con los requisitos, la calidad es alta, de lo contrario la calidad es baja. Una característica inherente que no cumple con los requisitos correspondientes es una no conformidad.

Para medir las no conformidades de un tesauro es conveniente contar con indicadores. Según la norma ISO 11620:2008 (International Organization for Standardization, 2008), un indicador es "una expresión (que puede ser numérica, simbólica o verbal) utilizada para caracterizar actividades (sucesos, objetos, personas) en términos cuantitativos y cualitativos, con el propósito de evaluar el valor de las actividades caracterizadas y el método asociado". Los indicadores son así herramientas que proveen un valor de referencia a partir del cual se puede establecer una comparación. En 1973, Orr estableció los criterios que definen un buen indicador, que luego fueron incorporadas a la norma ISO 11620:2008 (International Organization for Standardization, 2008; Orr, 1973). Éstos son los siguientes:

- Contenido informativo: el indicador tiene que ser informativo como herramienta para medir una actividad e identificar tanto logros como problemas y deficiencias, con el objetivo de poder actuar para remediarlos.

- Confiabilidad: el indicador debe producir el mismo resultado cuando se utiliza repetidamente bajo las mismas condiciones o circunstancias.

- Validez: el indicador debe medir lo que se quiere medir.

- Adecuación: el indicador debe ser acorde con el objetivo para el que se ha formulado. Las unidades, escalas y operaciones necesarias para implementar el proceso de medición deben ser apropiadas.

- Aplicabilidad: el indicador debe ser práctico, es decir debe utilizar datos que resulten accesibles, con un esfuerzo razonable en términos de tiempo y costos operativos.

- Comparabilidad: el indicador debe permitir que la calidad de dos o más actividades pueda ser comparada. En el caso del presente trabajo, debe permitir la comparación de la calidad de dos o más tesauros.

La experiencia más conocida para la evaluación de tesauros usando indicadores es la realizada por el Bureau Marcel Van Dijk (BMD) de Bruselas, que en 1976 llevó a cabo un estudio sobre 10 tesauros monolingües y 10 multilingües (Commission des Communautés Européenes, 1976). El BMD propuso varios indicadores que, al igual que el presente trabajo, se orientaban a la etapa de diseño y desarrollo, ya que incluso recomendaban su inclusión en la norma internacional. Otros autores han aplicado o recomendado estos indicadores (Gil Leiva, 2008; Álvaro Bermejo y otros, 1989; Gil Urdiciain, 1998), aunque Lancaster (2002) llegó a la conclusión de que si bien algunos eran ingeniosos, otros resultaban triviales e incluso arbitrarios. Por otro lado, hay que tener en cuenta que el BMD 
basó su evaluación en la primera edición de la norma ISO 2788:1974, que fue actualizada en 1986 y se encuentra actualmente en revisión (International Organization for Standardization, 2010).

El indicador del BMD que se ocupa de las relaciones jerárquicas es la tasa de enriquecimiento, definida como $A / B$, donde $A$ es la suma de relaciones jerárquicas y asociativas del tesauro y $B$ es el número de términos preferentes. El valor de referencia recomendado por el BMD fue de 2 a 5 relaciones por término preferente. En nuestra opinión, este indicador es cuestionable por los siguientes motivos: a) las relaciones jerárquicas y las asociativas cumplen distintas funciones en el tesauro, pues mientras que las relaciones jerárquicas son obligatorias para todos los términos preferentes, las asociativas solo se aplican en algunos casos, entonces las exigencias en cuanto a su inclusión en el tesauro son distintas y no deberían sumarse; $b$ ) es difícil aceptar sin mayores argumentos el valor de referencia de 2 a 5 relaciones por término preferente para todos los tesauros de todos los dominios; c) el indicador no aporta información sobre el correcto empleo de las relaciones jerárquicas y de las relaciones asociativas.

En consecuencia, el propósito del presente trabajo es proponer indicadores que permitan medir las no conformidades en la estructura sistemática de los tesauros, respecto tanto a las normas para su diseño y desarrollo, como a los principios de la teoría de la clasificación.

\section{Material y métodos}

En primer lugar, aquí se considera que los requisitos son las especificaciones establecidas por las normas para el diseño y desarrollo de tesauros, así como los principios de la teoría de la clasificación, mientras que las características inherentes son los rasgos que presentan los tesauros evaluados.

Las normas tomadas en cuenta para identificar los requisitos son las siguientes:

- ISO 2788:1986 para tesauros monolingües. Esta norma está vigente, pero en revisión, ya que una nueva norma ISO 25964 se encuentra en preparación y reemplazará a la anterior (International Organization for Standardization, 1986, 2010).

- ANSI/NISO Z39.19:2005. Es la cuarta edición de la norma estadounidense y ha sido modificada para incluir otros sistemas de organización del conocimiento. Aquí se toman en cuenta solamente las especificaciones pertinentes a los tesauros (National Information Standards Organization, 2005).

- BS 8723-2:2005. Es la tercera edición de la norma británica y al igual que la estadounidense incluye otros sistemas de organización de conocimiento. El tesauro está tratado en la Parte 2, que ha sido tomada en cuenta para este trabajo (BSI Group, 2005a). 
También se han tenido en consideración los principios de la teoría de la clasificación postulados por diferentes autores que se mencionan más adelante.

Se proponen los siguientes indicadores, cuyos valores de referencia se discuten en la sección Resultados y discusión:

1. Porcentaje de términos preferentes que designan más de un concepto. Se define como $A / B \times 100$, donde $A$ es el número de términos preferentes que designan más de un concepto y $B$ es el número total de términos preferentes.

2. Porcentaje de términos preferentes que carecen de relación jerárquica (términos huérfanos). Se define como $A / B \times 100$, donde $A$ es el número de términos preferentes que carecen de relación jerárquica y $B$ es el número total de términos preferentes.

3. Porcentaje de términos preferentes con dos o más términos genéricos (TG). Se define como $A / B \times 100$, donde $A$ es la suma de términos preferentes que poseen dos o más TG y $B$ es el número total de términos preferentes.

4. Porcentaje de términos preferentes con un solo término específico (TE). Se define como $A / B \times 100$, donde $A$ es la suma de términos preferentes que poseen un solo TE y $B$ es el número total de términos preferentes.

Para probar estos indicadores, se llevó a cabo una prueba de aplicación en una muestra de términos preferentes tomados de tres tesauros en español:

- Descriptores en ciencias de la salud (DeCS): especializado en ciencias de la salud. Es una adaptación del MeSH, desarrollado por BIREME-Centro Latinoamericano y del Caribe en Ciencias de la Salud (BIREME, 2008).

- Tesauro Spines (Spines): es un tesauro especializado en ciencia y tecnología, desarrollado por la Unesco (Organización de las Naciones Unidas para la Educación, la Ciencia y la Cultura, 1988).

- Tesauro de la Unesco (Unesco): es también un tesauro desarrollado por la Unesco sobre educación, ciencia y cultura (Organización de las Naciones Unidas para la Educación, la Ciencia y la Cultura, 2007).

La prueba de aplicación se realizó sobre una muestra al azar de cada uno de los tesauros mencionados (nivel de confianza 95\%, intervalo de confianza 5), resultando 379 términos preferentes para DeCS, 370 para Spines y 368 para Unesco. No se incluyeron nombres propios en la muestra. 


\section{Resultados y discusión}

Los resultados de la prueba de aplicación se muestran en la tabla I.

\section{TABLA I}

Resultados obtenidos en la prueba de aplicación de los indicadores propuestos a los tesauros evaluados

\begin{tabular}{l|c|c|c}
\hline \multicolumn{1}{c|}{ Indicador } & DeCS \% & Spines \% & Unesco \% \\
\hline $\begin{array}{l}\text { 1. Porcentaje de términos preferentes que representan } \\
\text { más de un concepto. }\end{array}$ & 10,8 & 7,3 & 10,9 \\
\hline $\begin{array}{l}\text { 2. Porcentaje de términos preferentes que carecen de } \\
\text { relación jerárquica. }\end{array}$ & 0 & 1,4 & 1,1 \\
\hline $\begin{array}{l}\text { 3. Porcentaje de términos preferentes con dos o más } \\
\text { términos genéricos. }\end{array}$ & 53,8 & 14,9 & 0 \\
\hline $\begin{array}{l}\text { 4. Porcentaje de términos preferentes con un solo tér- } \\
\text { mino específico. }\end{array}$ & 13,5 & 3,5 & 10,3 \\
\hline
\end{tabular}

\subsection{Porcentaje de términos preferentes que representan más de un concepto}

El valor de referencia para este indicador es 0 , ya que las normas para el diseño y desarrollo de tesauros especifican que un término preferente debe designar un y solo un concepto, e inversamente un concepto debe ser designado por un y solo un término preferente (International Organization for Standardization, 1986; National Information Standards Organization, 2005; BSI Group, 2005a). Así, los tres tesauros evaluados muestran numerosas no conformidades respecto a este requisito. Algunos ejemplos se presentan en la tabla II.

Como se puede observar, algunos tesauros cuentan con términos preferentes que pueden poscoordinarse. Por ejemplo el Tesauro de la Unesco posee un término preferente recursos financieros y otro término preferente educación, cada uno de los cuales designa un solo concepto. Entonces, no se justifica la inclusión de un tercer término preferente que combina los dos conceptos anteriores como recursos financieros en educación, porque al momento de realizar una búsqueda en una base de datos bibliográfica se pueden poscoordinar "recursos financieros" AND educación. Por lo tanto, si en la ecuación de búsqueda se emplean estos dos últimos términos preferentes, se perderán los registros indizados por el término preferente recursos financieros en educación. Casos similares a éste se presentan en los tres tesauros analizados.

Por otro lado, es interesante recordar la llamada teoría analítica del concepto orientada al referente postulada por Ingetraut Dahlberg (1995), que se esquematiza en el triángulo del concepto (figura 1). 


\section{TABLA II}

Ejemplos de términos preferentes que designan más de un concepto, tomados de los tesauros DeCS, Spines y Unesco

\begin{tabular}{c|l|l}
\hline \multicolumn{1}{c}{ Tesauro } & \multicolumn{1}{c}{ Término preferente } & \multicolumn{1}{c}{ Forma correcta } \\
\hline \multirow{3}{*}{ DeCS } & virus de la encefalitis de California & $\begin{array}{l}\text { virus* } \\
\text { encefalitis de California* }\end{array}$ \\
\cline { 2 - 3 } & economía de la salud & $\begin{array}{l}\text { economía* } \\
\text { salud* }\end{array}$ \\
\hline \multirow{2}{*}{ Spines } & piezas moldeadas en arena & $\begin{array}{l}\text { piezas moldeadas* } \\
\text { arena* }\end{array}$ \\
\cline { 2 - 3 } & legislación sobre medicamentos & $\begin{array}{l}\text { legislación* } \\
\text { productos farmacéuticos (UP medica- } \\
\text { mentos) }\end{array}$ \\
\hline \multirow{2}{*}{ Unesco } & salario del profesor & $\begin{array}{l}\text { salario* } \\
\text { docente* (UP profesor) }\end{array}$ \\
\cline { 2 - 3 } & recursos financieros en educación & $\begin{array}{l}\text { educación* } \\
\text { recursos financieros* }\end{array}$ \\
\hline
\end{tabular}

\footnotetext{
* Términos preferentes existentes en los tesauros respectivos. UP: usado por.
}

FIGURA 1

Triángulo del concepto de Dablberg (20)

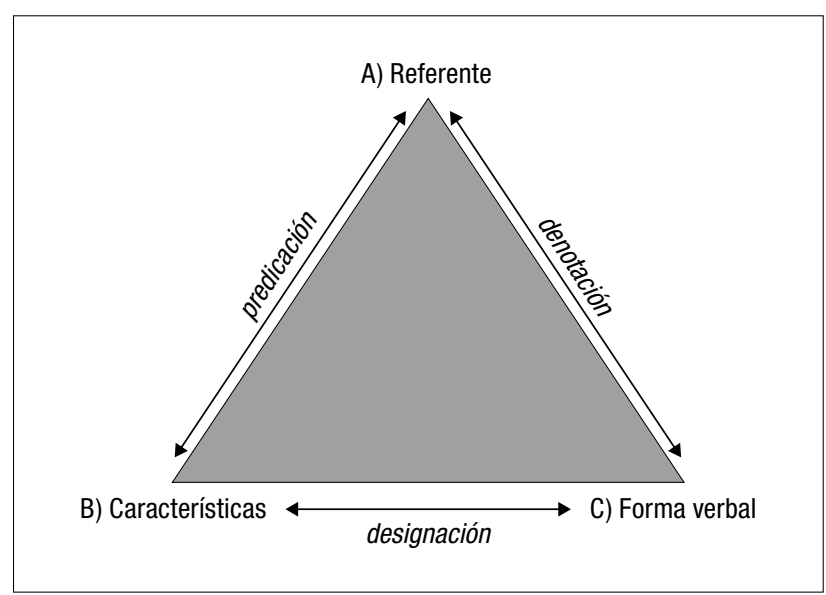

El referente es un ítem de referencia al universo de ideas, objetos, hechos, leyes, propiedades, acciones, etc. Cuando mentalmente se alude a un referente, mediante una declaración o predicación acerca del mismo, se demuestra aquello que se conoce acerca de ese referente y se generan, mediante esta demostración, 
los elementos del concepto que se están formulando a través de tales declaraciones. Las declaraciones verdaderas y esenciales acerca del referente son elementos de conocimiento o características del concepto. Por eso Dahlberg considera que el concepto es una unidad de conocimiento y no una unidad de pensamiento como definen otras fuentes (International Organization for Standardization, 1986; National Information Standards Organization, 2005; BSI Group, 2005a). Esta unidad de conocimiento o concepto, construido por declaraciones verdaderas y esenciales de un referente, se sintetiza en una designación verbal (término) o simbólica (notación). En un tesauro, las características del concepto permiten definirlo y también subdividirlo, lo cual conduce justamente a la estructura sistemática del tesauro. Considerando esta afirmación, no deberían incluirse frases que combinen dos o más conceptos, como por ejemplo el término preferente informática y desarrollo del Tesauro de la Unesco, porque ¿cuál de los dos conceptos aporta las características a considerar para el proceso de subdivisión que permita obtener las relaciones jerárquicas?

El vínculo concepto-término es importante además para la interoperabilidad entre dos o más tesauros o entre un tesauro y otro sistema de organización del conocimiento (SOC). Como señalan las nuevas normas estadounidense y británica (National Information Standards Organization, 2005; BSI Group, 2007), el punto crítico de la interoperabilidad entre SOC es la equivalencia entre términos preferentes, pero esa equivalencia se facilita cuando cada término preferente, sea cual fuere, representa un solo concepto. Así, un tesauro que incluya el término preferente manies, podrá mapearse sin dificultad con otros tesauros que incluyan ese mismo concepto, aunque esté designado por otros términos preferentes como cacahuates, cacabuetes, peanuts o amendoims.

Coinciden con lo anterior las recomendaciones del Consorcio World Wide Web (W3C) para los SOC simples (SKOS) y la asignación de un identificador de recurso uniforme (URI) a cada concepto, de modo que puedan mapearse entre distintos SKOS, tanto los términos preferentes como los no preferentes en cualquier idioma (W3C Working Group, 2009).

\subsection{Porcentaje de términos preferentes que carecen de relación jerárquica}

El valor de referencia para este indicador es 0 , ya que las normas para el diseño y desarrollo de tesauros especifican que un término preferente debe tener al menos una relación jerárquica (International Organization for Standardization, 1986; National Information Standards Organization, 2005; BSI Group, 2005a). Aquellos términos preferentes que se encuentran en el nivel más alto de la jerarquía tendrán solamente TE, mientras que los que ocupan el nivel más bajo de la jerarquía tendrán solamente TG. Todos los términos preferentes que se hallan en los niveles intermedios tendrán TG y TE. De modo que no puede haber un solo término preferente huérfano, es decir sin relación jerárquica. Tanto Spines 
(5 términos huérfanos) como Unesco (4 términos huérfanos) mostraron no conformidades para este requisito.

\subsection{Porcentaje de términos preferentes con dos o más términos genéricos}

En su primera parte, la nueva norma británica para el diseño y desarrollo de vocabularios estructurados BS 8723-1:2005 (BSI Group, 2005b) considera que la estructura sistemática puede ser monojerárquica o polijerárquica. En la estructura monojerárquica, cada concepto tiene un solo concepto genérico. Aplicado al tesauro, significa que un término preferente solo puede tener un TG. Este enfoque coincide con el principio de lugar de definición única postulado por Farradane (1952) y revalorado recientemente por otros autores (Gnoli, 2007). Por el contrario, en la estructura polijerárquica un concepto puede tener más de un concepto genérico, es decir un término preferente podría mostrar más de un TG. Este enfoque coincide con las especificaciones de las normas para el diseño y desarrollo de tesauros, aunque cabe aclarar que estas normas indican claramente que la relación polijerárquica solo ocurre "en algunos casos" (International Organization for Standardization, 1986; National Information Standards Organization, 2005; BSI Group, 2005a). Una discusión sobre este tema se puede encontrar en el artículo de Miller (1997).

Si se aplica el concepto de estructura monojerárquica, entonces el valor de referencia de este indicador es 0 , ya que ningún término preferente puede tener más de un TG. En tal caso, tanto DeCS como Spines muestran un importante número de no conformidades.

Si se aplica el concepto de estructura polijerárquica no hay un valor de referencia, pero debe recordarse que las normas aceptan la relación polijerárquica solo "en algunos casos" y con un fundamento lógico. A continuación se analizan y comparan dos casos:

El primer caso es un ejemplo típico de relación polijerárquica que aparece en todas las normas analizadas (International Organization for Standardization, 1986; National Information Standards Organization, 2005; BSI Group, 2005a). Se trata del instrumento musical órgano, que puede ser un instrumento de teclado y al mismo tiempo un instrumento de viento. Se mostrarían entonces las siguientes relaciones:

órganos

TG instrumentos de teclado

TG instrumentos de viento

Cuando se analiza cada relación, se descubre que son relaciones jerárquicas de tipo género/especie, es decir el órgano es una especie de instrumentos de teclado y al mismo tiempo una especie de instrumento de viento, hay en esto un fundamento lógico. 
El segundo caso es el término preferente secretina, tomado del DeCS, que posee cinco TG, según las siguientes jerarquías:

\title{
hormonas
}

hormonas gastrointestinales

\section{secretina}

\author{
hormonas \\ hormonas peptídicas \\ secretina \\ péptidos \\ neuropéptidos \\ secretina \\ péptidos \\ hormonas peptídicas \\ secretina \\ proteínas del sistema nervioso
neuropéptidos
secretina
}

¿Qué es entonces la secretina: una hormona gastrointestinal, una hormona peptídica, un neuropéptido? ¿Qué es una hormona peptídica: una hormona, un péptido o una proteína? La definición que aporta el propio DeCS para secretina es: "hormona peptídica de 27 aminoácidos de la mucosa duodenal, que activa la secreción pancreática y disminuye los niveles sanguíneos de azúcar". DeCS también aporta una definición de hormonas peptídicas: "hormonas sintetizadas de aminoácidos. Se distinguen de los péptidos y proteínas de señalización intercelular en que sus acciones son sistémicas". Entonces, si la secretina es una hormona peptídica y las hormonas peptídicas son hormonas, la única de las jerarquías mencionadas anteriormente que tiene un fundamento lógico, por ser una relación jerárquica de tipo género/especie, es la siguiente:

\section{hormonas \\ hormonas peptídicas \\ secretina}

La indicación en el orden alfabético del tesauro podría ser:

secretina

TG hormonas peptídicas

TR hormonas gastrointestinales

TR neuropéptidos 
En consecuencia, si bien no se puede recomendar un valor de referencia, consideramos que un número alto de TG para un solo término preferente debería llamar la atención del evaluador, al menos para verificar si las relaciones jerárquicas tienen realmente un fundamento lógico, es decir si son relaciones jerárquicas de cualquiera de los tres tipos especificados por las normas: género/especie, todo/parte o enumerativas (International Organization for Standardization, 1986; National Information Standards Organization, 2005; BSI Group, 2005a).

\subsection{Porcentaje de términos preferentes con un solo término específico}

El valor de referencia para este indicador es 0 , ya que en un tesauro no puede haber un término preferente con un solo TE. Como se indicó anteriormente, un concepto puede ser subdividido por alguna de sus características, para generar conceptos más específicos. Aunque las normas para el diseño y desarrollo de tesauros no lo mencionan, Ranganathan postuló el llamado canon de diferenciación, según el cual una característica usada para subdividir un concepto debe generar al menos dos conceptos más específicos y mutuamente excluyentes, pues de lo contrario no hay subdivisión (Ranganathan, 1967; Spiteri, 1998). De modo que los términos preferentes de un tesauro deben tener, al menos, dos TE, con excepción de aquellos que ocupan el último nivel de la jerarquía, que carecen de TE. En la prueba de aplicación, los tres tesauros evaluados mostraron no conformidades respecto a este requisito. Algunos ejemplos se muestran en la tabla III.

\section{TABLA III}

Ejemplos de términos preferentes con un solo término específico

\begin{tabular}{l|l|l}
\hline \multirow{4}{*}{ Tesauro } & \multicolumn{1}{|c}{ Término preferente } & \multicolumn{1}{c}{ Único término específico } \\
\hline \multirow{4}{*}{ DeCS } & papaverina & tetrahidropapaverolina \\
\cline { 2 - 3 } & oxalatos & oxalato de calcio \\
\cline { 2 - 3 } & oncología médica & oncología por radiación \\
\hline \multirow{4}{*}{ Spines } & audioelectrónica & síntesis de la palabra \\
\cline { 2 - 3 } & galaxias & Vía Láctea \\
\cline { 2 - 3 } & pensamiento creativo & aptitud creativa \\
\hline \multirow{3}{*}{ Unesco } & paisaje cultural & sitio sagrado \\
\cline { 2 - 3 } & magia & brujería \\
\cline { 2 - 3 } & onda de radio & microondas \\
\hline
\end{tabular}




\section{Conclusiones}

Se considera importante replantear el uso de indicadores para la evaluación de tesauros. En este sentido, se proponen aquí cuatro indicadores, aplicables a la etapa de diseño y desarrollo del tesauro, que contribuyen a evaluar la calidad de su estructura sistemática, antes de que sea utilizado por los futuros indizadores y usuarios de la información. Es importante resaltar que estos indicadores contribuyen a la evaluación, es decir pueden complementarse con otros métodos de evaluación cuantitativos y cualitativos.

Los cuatro indicadores propuestos en este trabajo cumplen con los criterios de Orr (1973) en cuanto a ser informativos, válidos, adecuados y comparables. Respecto a la confiabilidad, si bien han sido aplicados a tres tesauros distintos con resultados equivalentes, sería necesaria su reiteración en otras experiencias, por otras personas, sobre otros tesauros y durante un cierto tiempo, para poder afirmar que son verdaderamente confiables. En cuanto a la aplicabilidad, los indicadores 2, 3 y 4 son muy prácticos, ya que pueden incorporarse fácilmente a cualquier software de gestión de tesauros, de tal modo que el diseñador pueda ser alertado por el propio software de posibles no conformidades. El indicador 1 , si bien demanda mayor intervención humana, solo requiere un esfuerzo razonable, particularmente si se tiene en cuenta que el objetivo final es evaluar la calidad de la estructura sistemática del tesauro.

Es importante destacar que tanto los requisitos contra los cuales se deben comparar las características de los tesauros, como las pautas para evaluar la calidad de cualquier objeto, proceso o sistema, están especificados en normas nacionales e internacionales, ampliamente conocidas, aceptadas y fáciles de consultar. De modo que la tarea de establecer indicadores y evaluar la calidad de los distintos aspectos del tesauro, como en este caso la estructura sistemática, debe basarse en dichos requisitos documentados.

\section{Agradecimientos}

Proyecto acreditado PID-UNLP H004 de la Universidad Nacional de La Plata.

\section{Bibliografía}

Aitchison, J.; Gilchrist, A., y Bowen, D. (2000). Thesaurus construction and use: a practical manual (4. ${ }^{\mathrm{a}}$ ed). London: Aslib, 47-80.

Alvaro Bermejo, C.; Villagra Rubio, A., y Sorli Rojo A. (1989). Desarrollo de lenguajes documentales formalizados en lengua española: II. Evaluación de los tesauros disponibles en lengua española. Revista Española de Documentación Científica, vol. 12 (4), 283-305.

Bireme: Centro Latinoamericano y del Caribe en Ciencias de la Salud. (2008). Descriptores 
en ciencias de la salud [en línea]. São Paulo: El Centro, http://decs.bvs.br/E/homepagee.htm [consulta: 23-12-2010].

BSI Group. (2005a). Structured vocabularies for information retrieval: guide. Part 2: thesauri. London: The Group (BS 8723-2:2005).

BSI Group. (2005b). Structured vocabularies for information retrieval: guide. Part 1. Definitions, symbols and abbreviations. London: The Group (BS 8723-1:2005).

BSI Group. (2007). Structured vocabularies for information retrieval: guide. Part 4. Interoperability between vocabularies. London: The Group, (BS 8723-4:2007).

Chung, Y. M., y Lee, J. Y. (2004). Optimization of some factors affecting the performance of query expansion. Information Processing and Management, vol. 40 (6), 891-917.

Claverdon, C. W. y Mills, J. (1985). The testing of index language devices. En: Chan, L. M.; Richmond P. A., y Svenonius, E. (eds.). Theory of subject analysis: a sourcebook. Littleton: Libraries Unlimited, 221-246.

Commission des Communautés Européennes. (1976). Définition des charactéristiques essentielles des thesauri: Rapport final. Bruxelles: Bureau Marcel van Dijk, vol. 1.

Dahlberg, I. (1995). Current trends in knowledge organization. En: García Marco, F. J. (ed.). Actas del I Encuentro ISKO-España. Organización del Conocimiento en Sistemas de Información y Documentación, vol. 1, 7-25.

Farradane, J. E. L. (1952). A scientific theory of classification and indexing: further considerations. Journal of Documentation, vol. 8 (2), 73-92.

Foskett, A. C. (1996). Subject approach to information (5. ${ }^{a}$ ed). London: Library Association, $12-32$.

Gil Leiva, I. 2008. Manual de indización: teoría y práctica. Gijón: Trea, 213-217.

Gil Urdiciain, B. (1998). Evaluación semántica y estructural de tesauros. Revista General de Información y Documentación, vol. 8 (2), 193-199.

Gnoli, C. (2007). Progress in synthetic classification: towards unique definitions of concepts [en línea]. En: UDC Seminar 4 -5 June 2007: The Hague: UDC Consortium.

International Organization for Standardization. 1986. Documentation: guidelines for the establishment and development of monolingual thesauri (2. ${ }^{\mathrm{a}}$ ed.). Geneva: The Organization. (ISO 2788:1986).

International Organization for Standardization. (2000). Quality management systems: fundamentals and vocabulary ( $2^{a}$ ed.). Geneva: The Organization. Geneva: The Organization. (ISO 9000:2000).

International Organization for Standardization. (2008). Information and documentation: library performance indicators. Geneva: The Organization. (ISO 11620:2008).

International Organization for Standardization. (2010). Information and documentation: Thesauri and interoperability with other vocabularies: Part 1. Thesauri for information retrieval [standard under development]. Geneva: The Organization. (ISO/DIS 259641:2010).

Lancaster, F. W. (2002). Control del vocabulario para la recuperación de información (2. ${ }^{a}$ ed). Valencia: Università de Valencia. 31-37, 55-62.

Miller, U. (1997). Thesaurus construction: problems and their roots. Information Processing and Management, vol. 33 (4), 481-493. 
Mills, J. (2004). Faceted classification and logical division in information retrieval. Library Trends, vol. 52 (3), 541-570.

National Information Standards Organization (2005). Guidelines for the construction, format, and management of monolingual controlled vocabularies [en línea]. Bethesda MD; NISO Press (ANSI/NISO Z39.19:2005). http://www.niso.org/standards/resources/ Z39-19-2005.pdf [consulta: 23-12-2010].

Organización de las Naciones Unidas para la Educación, la Ciencia y la Cultura (1988). Tesauro Spines [en línea]. París: La Organización. http://thes.cindoc.csic.es/index_SPIN_ esp.html [consulta: 23-12-2010].

Organización de las Naciones Unidas para la Educación, la Ciencia y la Cultura (2007). Tesauro de la Unesco (15. a ed.). París: La Organización. http://databases.unesco.org/ thessp/ [consulta: 23-12-2010].

Orr, R. H. (1973). Measuring the goodness of library services: a general framework for considering quantitative measures. Journal of Documentation, vol. 29 (3), 315-332.

Owens, L. A., y Cochrane, P. A. (2004). Thesaurus evaluation. En: Roe, S. K., y Thomas, A. R. (eds.). The thesaurus: review, renaissance, and revision. New York: Haworth Information Press, 87-102.

Ranganathan, S. R. (1967). Prolegomena to library classification (3. ${ }^{\mathrm{a}}$ ed.). New York: Asia Publishing House, Chapter EC, 145.

Spiteri, L. A. (1998). Simplified model for facet analysis. Canadian journal of information and library science, vol. 23 (1), 1-30.

Vickery, B. C. (1960). Faceted classification: a guide to the construction and use of special schemes. London: Aslib.

W3C Working Group (2009). SKOS simple knowledge organization system primer [en línea]. s.L.: W3C. Disponible en la World Wide Web http://www.w3.org/TR/2009/NOTE-skosprimer-20090818/\#secconcept [consulta 23-12-2010]. 